\title{
A CYLINDRICAL WAVE-MAKER PROBLEM IN A LIQUID OF FINITE DEPTH WITH AN INERTIAL SURFACE IN THE PRESENCE OF SURFACE TENSION
}

\author{
N. K. GHOSH
}

(Received 12 June 1990; revised 23 August 1990)

\begin{abstract}
The problem of generation of waves in a liquid of uniform finite depth with an inertial surface composed of a thin but uniform distribution of disconnected floating particles, due to forced axisymmetric motion prescribed on the surface of an immersed vertical cylindrical wave-maker of circular cross section under the influence of surface tension at the inertial surface, is discussed. The techniques of Laplace transform in time and the modified Weber transform involving Bessel functions in the radial coordinate have been employed to obtain the velocity potential. The steady-state development to the potential function as well as the inertial surface depression due to time-harmonic forced oscillations of the wave-maker are deduced. It is found that the presence of surface tension at the inertial surface ensures the propagation of time-harmonic progressive waves of any angular frequency.
\end{abstract}

\section{Introduction}

Problems of forced two-dimensional wave motion with outgoing surface waves at infinity, generated by a harmonically oscillating vertical plane or circular cylinder immersed in deep water, were solved by Havelock [3] long ago within the framework of linear theory of water waves. The cylindrical wave-maker problem is of some physical relevance, as offshore structures in high seas for the purpose of oil prospecting are usually constructed in the form of vertical cylindrical columns. Recently Mandal [5] reinvestigated Havelock's [3] cylindrical wave-maker problem in water of both infinite and finite depths by applying the modified Weber transform in radial

\footnotetext{
'Department of Applied Mathematics, Calcutta University, Calcutta-700009, India.

(C) Copyright Australian Mathematical Society 1991, Serial-fee code 0334-2700/91
} 
coordinate. Earlier Rhodes-Robinson [9] extended this problem, making allowance for surface tension at the free surface, wherein the unique solution of the boundary-value problem for a time-harmonic velocity potential describing the motion was obtained by assuming the free-surface slope at the wave-maker to be a time-harmonic oscillation of prescribed amplitude. The condition at the edge where the free surface meets the obstacles was first assumed by Evans [1, 2] while considering transmission and reflection of incoming progressive waves by a vertical barrier and forced waves due to the heaving waves by a vertical barrier and forced waves due to the heaving motion of a half immersed circular cylinder in deep water. However, this type of edge condition may not be realistic and is a matter of considerable research interest in recent times (cf. Hocking [4] and others).

Problems concerning generation of surface waves in a liquid covered by an inertial surface composed of a thin uniform distribution of noninteracting floating materials (e.g. broken ice, unstretched mat) were considered recently in a number of papers [6-8, 9-11]. Rhodes-Robinson [10] also mentioned how the plane vertical wave-maker problem can be solved for a liquid with an inertial surface. Using a Laplace transform in time, the associated initialvalue problem was reduced to a boundary-value problem for the transformed potential function. Then Green's integral theorem was applied to a suitably defined Green's function and this transformed potential to solve the problem. However, the cylindrical wave-maker problem in a liquid with an inertial surface cannot be solved by this method. For a liquid of both infinite and finite uniform depths, this problem was solved by Mandal and Kundu [7, 8] by employing the modified Weber transform, after taking Laplace transform in time.

In the present paper, we consider the cylindrical wave-maker problem in a liquid of uniform finite depth with an inertial surface in the presence of surface tension, wherein the edge condition is assumed to be similar to that used by Rhodes-Robinson [10] for a vertical wave-maker. The edge condition assumed in [10], although not physically realistic, is used here to demonstrate mathematically how the known solution of the cylindrical wave-maker problem for a liquid of finite depth with a free surface in the presence of surface tension considered in [9] can be extended to a liquid with an inertial surface. A modified Weber transform used earlier in $[5,7]$ has been employed herein to obtain the transformed potential function after taking Laplace transform in time. Laplace inversion then produces the velocity potential. For the consideration of time-harmonic case we assume that the wave-maker and the prescribed inertial surface slope at the wave-maker have the same frequency. The steady-state development to the potential function and the inertial surface depression are obtained by following a procedure used in [10]. It is 
observed that the time-harmonic progressive waves generated by the wavemaker will propagate for any frequency at a large distance from it. When the surface density of the floating materials is made equal to zero, results for a liquid with a free surface are recovered. Putting $M=0$, results for a liquid with an inertial surface can also be deduced.

\section{Statement and formulation of the problem}

We formulate the problem for irrotational motion under the action of both gravity $g$ and surface tension $T$, say, of a liquid of density $\rho$ whose surface is covered by an inertial surface composed of a thin uniform distribution of disconnected floating matter of area density $\rho \varepsilon$. The case $\varepsilon=0$ corresponds to a liquid with a free surface. The normal fluid velocity on the surface of an immersed vertical circular cylindrical wave-maker extended throughout the depth of the liquid is supposed to be prescribed, and is both time and depth dependent. We choose a cylindrical coordinate system $(r, \theta, y)$ in which the $y$-axis is taken vertically downwards and is the axis of the cylindrical wave-maker with radius ' $a$ ' and the plane $y=0, r>a$ is the undistributed position of the inertial surface.

The motion of the liquid is axisymmetric, as the normal fluid velocity on the surface of the wave-maker is uniform in $\theta$. Since the motion starts from rest, it is irrotational and can be described by a velocity potential $\varphi(r, y ; t)$ satisfying the Laplace's equation

$$
\varphi_{r r}+\frac{1}{r} \varphi_{r}+\varphi_{y y}=0, \quad a<r<\infty, 0<y<h .
$$

If the inertial surface has depression $\zeta(r, t)$ from the equilibrium position, assuming linear theory, the joint boundary conditions relating to $\varphi$ and $\zeta$ are the kinematic condition

$$
\varphi_{y}=\zeta_{t} \text { on } y=0
$$

and the inertial surface condition

$$
\frac{\partial^{2}}{\partial t^{2}}\left(\varphi-\varepsilon \varphi_{y}\right)-g \varphi_{y}-\frac{T}{\rho} \varphi_{y y y}=0, \quad \text { on } y=0 .
$$

The condition at the wave-maker is

$$
\varphi_{r}=U_{1}(y, t) H(t) \text { on } r=a
$$

where $H(t)$ is the Heaviside unit step function and the bottom condition is

$$
\varphi_{y}=0, \quad \text { on } y=h .
$$


The initial conditions (cf. [10]) are

$$
\frac{\partial}{\partial t}\left(\varphi-\varepsilon \varphi_{y}\right)=\varphi-\varepsilon \varphi_{y}=0 \text { at } t=0, y=0,
$$

and lastly the edge condition describing the inertial surface slope at the wavemaker (cf. [10]) is

$$
\varphi_{r y}(a+, 0, t)=\pi \lambda^{\prime}(t)
$$

which is obtained from (2.2) after assuming $\zeta_{r}(a, t)=\pi \lambda(t)$ where $\lambda(t)$ is prescribed.

Let $\bar{\varphi}(r, y ; p)$ be the Laplace transforms of $\varphi(r, y ; t)$ defined by

$$
\bar{\varphi}=\int_{0}^{\infty} \varphi e^{-p t} d t \quad(p>0)
$$

Then $\bar{\varphi}$ satisfies the following boundary value problem:

$$
\left.\begin{array}{l}
\bar{\varphi}_{r r}+\frac{1}{r} \bar{\varphi}_{r}+\bar{\varphi}_{y y}=0, \quad a<r<\infty, \quad 0<y<h, \\
p^{2} \bar{\varphi}-\left(g+\varepsilon p^{2}\right) \bar{\varphi}_{y}-\frac{T}{\rho} \bar{\varphi}_{y y y}=0, \quad \text { on } y=0, \\
\bar{\varphi}_{r}=\bar{U}_{1}(y ; p) \text { on } r=a, \\
\bar{\varphi}_{y}=0 \text { on } y=h, \\
\bar{\varphi}_{r y}(a+, 0, p)=\pi p \bar{\lambda}(p)
\end{array}\right\}
$$

where $\bar{U}_{1}$ and $\bar{\lambda}_{1}$ are the Laplace transform of $U_{1}(y, t)$ and $\lambda(t)$ respectively.

\section{Solution by Weber transform}

To solve the boundary value problem (2.9) we note that a suitable transform formula in $(a, \infty)$ is to be used for removing the operator $\frac{\partial^{2}}{\partial r^{2}}+\frac{1}{r} \frac{\partial}{\partial r}$ in the first equation of (2.9) when $\bar{\varphi}_{r}$ is prescribed. This can be achieved if we use the Weber transform of a function $g(r)$ given in $(a, \infty)$, defined by

$$
\bar{g}(\xi)=\int_{a}^{\infty} r A(r, \xi) g(r) d r
$$

while the inverse transform formula is

$$
g(r)=\int_{0}^{\infty} \frac{\bar{g}(\xi) \xi A(r, \xi) d \xi}{J_{1}^{2}(a \xi)+Y_{1}^{2}(a \xi)}
$$

where

$$
A(r, \xi)=J_{1}(a \xi) Y_{0}(r \xi)-J_{0}(r \xi) Y_{1}(a \xi),
$$

$J_{n}, Y_{n}(n=0,1)$ being the Bessel functions of the first and second kinds respectively. 
The proof of (3.1) and (3.2) under suitable conditions imposed on $g(r)$, follows in the same manner as that given in [12] (where the Bessel functions of the same order $\nu>-\frac{1}{2}$ are used). This form of Weber transform has been used in $[5,7]$ in connection with a cylindrical wave-maker problem in a deep liquid.

Let $\Psi(\xi, y)$ be the Weber transform of $\bar{\varphi}(r, y)$ as defined by (3.1), then $\Psi(\xi, y)$ satisfies

$$
\left.\begin{array}{l}
\frac{d^{2} \Psi}{d y^{2}}-\xi^{2} \Psi=\frac{2}{\pi \xi} \bar{U}_{1}(y, p), \quad 0<y<h, \\
p^{2} \Psi-\left(g+\varepsilon p^{2}+T_{0} \xi^{2}\right) \Psi_{y}-2 T_{0} p \bar{\lambda}(p) / \xi=0, \quad \text { on } y=0
\end{array}\right\}
$$

where $T_{0} \equiv T / \rho$ and $\Psi_{y}=0$ on $y=h$. The solution of the BVP (3.4) is obtained as

$$
\Psi(\xi, y)=-\frac{2}{\pi}\left[\int_{0}^{h} G(y, s)\left\{\frac{\bar{U}_{1}(s, p)}{\xi}+\xi C(p)\right\} d s-\frac{C(p)}{\xi}\right]
$$

where $C(p) \equiv \pi T_{0} \bar{\lambda}(p) / p$ and $G(y, s)$ is the associated Green's function given by

$$
G(y, s)=\frac{\cosh \xi(h-s)}{\xi}\left[\frac{p^{2} \sinh \xi y+\xi\left(g+\varepsilon p^{2}+T_{0} \xi^{2}\right) \cosh \xi h}{p^{2} \cosh \xi h+\xi\left(g+\varepsilon p^{2}+T_{0} \xi^{2}\right) \sinh \xi h}\right], 0<y<s
$$

For $0<s<y$, we interchange $y$ and $s$ in the expression (3.6). Using the inverse Weber transform of (3.5) as defined by (3.2) we obtain after simplification

$$
\begin{aligned}
& \bar{\varphi}(r, y ; p)=- \frac{2}{\pi} \int_{0}^{\infty} \frac{A(r, \xi)}{J_{1}^{2}(a \xi)+Y_{1}^{2}(a \xi)} \\
& \times\left[\left\{\frac{\sinh \xi y+\varepsilon \xi \cosh \xi y}{D(\xi)}+\frac{\mu^{2} \cosh \xi(h-y)}{D(\xi) \sinh \xi h\left(p^{2}+\mu^{2}\right)}\right\}\right. \\
&\left.\cdot\left\{\int_{0}^{h} \frac{\cosh \xi(h-s) \bar{U}_{1}(s, p) d s}{\xi}+C(p) \sinh \xi h\right\}-C(p)\right] d \xi, \\
& 0<y<s
\end{aligned}
$$

where

$$
\left.\begin{array}{l}
D(\xi) \equiv \cosh \xi h+\varepsilon \xi \sinh \xi h, \quad M \equiv T_{0} / g \\
\mu^{2} \equiv \frac{g \xi\left(1+M \xi^{2}\right) \sinh \xi h}{D(\xi)}
\end{array}\right\}
$$


Replacing the Bessel functions involved in (3.7) in terms of the Hankel functions and taking Laplace inversion we obtain

$$
\begin{array}{r}
\varphi(r, y ; t) \\
=-\frac{1}{\pi i} \int_{0}^{\infty} B(r, \xi)\left[\frac { \operatorname { s i n h } \xi y + \varepsilon \xi \operatorname { c o s h } \xi y } { D ( \xi ) } \left\{\int_{0}^{h} \frac{\cosh \xi(h-s) \bar{U}_{1}(s, t)}{\xi} d s\right.\right. \\
\left.+C_{1}(t) \sinh \xi h\right\} \\
+\frac{\mu \cosh \xi(h-y)}{\xi D(\xi) \sinh \xi h} \int_{0}^{h} \cosh \xi(h-s)\left\{\int_{0}^{t} U_{1}(s, \tau) \sin \mu(t-\tau) d \tau\right\} d s \\
\left.+\pi T_{0} \mu \frac{\cosh \xi(h-y)}{D(\xi)}\left\{\int_{0}^{t} \sin \mu(t-\tau) d \tau \int_{0}^{\tau} \lambda(\alpha) d \alpha\right\}-C_{1}(t)\right] d \xi, \\
0<y<s,
\end{array}
$$

where

$$
\left.\begin{array}{l}
B(r, \xi) \equiv \frac{H_{0}^{(1)}(r \xi)}{H_{1}^{(1)}(a \xi)}-\frac{H_{0}^{(2)}(r \xi)}{H_{1}^{(2)}(a \xi)}, \\
C_{1}(t) \equiv \pi T_{0} \int_{0}^{t} \lambda(\tau) d \tau .
\end{array}\right\}
$$

Equation (3.9) is the general result for the potential function due to a vertical cylindrical wave-maker with outward normal fluid velocity $U_{1}(y, t)$ prescribed on its boundary together with a suitable prescribed downward inertial surface slope $\pi \lambda(t)$ at the wave-maker. The inertial surface depression at any time is obtained as

$$
\zeta(r, t)=\frac{1}{g} \frac{\partial}{\partial t}\left(\varphi-\varepsilon \varphi_{y}\right)(r, 0 ; t)
$$

where $\varphi$ is given by (3.9).

\section{Time-harmonic case and steady-state development}

For the time-harmonic case, we put $U_{1}(y, t)=U(y) \sin \sigma t$ and $\lambda(t)=$ $\sin \sigma t$ (cf. [10]) in (3.9), then (3.9) becomes 


$$
\begin{array}{r}
\varphi(r, y ; t) \\
=-\frac{1}{\pi i} \int_{0}^{\infty} B(r, \xi)\left[\frac{\sinh \xi y+\varepsilon \xi \cosh \xi y}{D(\xi)}\left\{\frac{a(\xi) \sin \sigma t}{\xi}+C_{2}(t) \sinh \xi h\right\}\right. \\
+a(\xi) \frac{\mu \cosh \xi(h-y)}{\xi D(\xi) \sinh \xi h} \frac{\mu \sin \sigma t-\sigma \sin \mu t}{\mu^{2}-\sigma^{2}} \\
\left.+\frac{\pi T_{0}}{\sigma} \frac{\cosh \xi(h-y)}{D(\xi)}\left\{1+\frac{\sigma^{2} \cos \mu t-\mu^{2} \cos \sigma t}{\mu^{2}-\sigma^{2}}\right\}-C_{2}(t)\right] d \xi
\end{array}
$$

where

$$
a(\xi) \equiv \int_{0}^{h} \cosh \xi(h-s) U(s) d s
$$

and

$$
C_{2}(t) \equiv\left(T_{0} \pi / \sigma\right)(1-\cos \sigma t)
$$

To obtain the steady-state development in (4.1), we must isolate and eliminate the transient terms. We note that $\mu^{2}-\sigma^{2}$ or equivalently $P(\xi)=$ $\xi\left(1-\varepsilon K+M \xi^{2}\right) \sinh \xi h-K \cosh \xi h$ (where $K \equiv \sigma^{2} / g$ ) in the denominator of the integral involving $\sin \mu t$ or $\cos \mu t$ vanishes in the range of integration $\xi>0$ for all $\varepsilon K \geq 0$ and $M>0$. For $M>0$ and $\varepsilon K \geq 0, P(\xi)$ has always two real zeros (cf. [9]), one positive and another negative, $k_{0}$ and $-k_{0}$ say, and an infinite number of imaginary zeros $\pm i k_{n}$ say, where $k_{n}$ 's $(n=1,2, \ldots)$ are real and positive and satisfy the equation

$$
k_{n}\left(1-\varepsilon K-M k_{n}^{2}\right) \sin k_{n} h+K \cos k_{n} h=0 .
$$

We now introduce a Cauchy principal value at $\xi=k_{0}$ and follow a procedure as used in [10]. Then the integral involving $\sin \mu t$ in (4.1) is

$$
\begin{aligned}
\frac{\sigma}{\pi i} \int_{0}^{\infty} \frac{B(r, \xi) a(\xi) \cosh \xi(h-y)}{\xi D(\xi) \sinh \xi h} \frac{\sin \mu t}{\mu^{2}-\sigma^{2}} d \xi \\
=\frac{4 \sigma}{\pi i} \int_{0}^{\infty}\left[\frac{B\left(r, \xi^{\prime}\right) a\left(\xi^{\prime}\right)\left(1+M \xi^{\prime 2}\right) \cosh \xi^{\prime}(h-y)}{\left\{\left(1+3 M \xi^{\prime 2}\right) \sinh 2 \xi^{\prime} h+2 \xi^{\prime} h\left(1+M \xi^{\prime 2}\right)+4 M \varepsilon \xi^{\prime^{\prime 3}} \sinh ^{2} \xi^{\prime} h\right\}\left(\mu\left(\xi^{\prime}\right)+\sigma\right)}\right]_{\xi^{\prime}=k_{0}}^{\xi} \\
\quad \times \frac{\sin \mu t}{\mu-\sigma} d \mu \\
+\frac{2}{\pi i}\left[\frac{B\left(r, k_{0}\right) a\left(k_{0}\right)\left(1+M k_{0}^{2}\right) \cosh k_{0}(h-y)}{\left(1+3 M k_{0}^{2}\right) \sinh 2 k_{0} h+2 k_{0} h\left(1+M k_{0}^{2}\right)+4 M e k_{0}^{3} \sinh ^{2} k_{0} h}\right] f_{0}^{\infty} \frac{\sin \mu t}{\mu-\sigma} d \mu
\end{aligned}
$$

where the last integral is in the sense of Cauchy principal value. Now as $t \rightarrow \infty$, the first term of (4.4) vanishes by the application of the RiemannLebesgue lemma and the second term tends to $\pi \cot \sigma t$ as $t \rightarrow \infty$. Thus as 
$t \rightarrow \infty,(4.4)$ becomes

$$
-2 i \cos \sigma t\left[\frac{B\left(r, k_{0}\right) a\left(k_{0}\right)\left(1-\varepsilon K+M k_{0}^{2}\right) \cosh k_{0}(h-y)}{\left(1-\varepsilon K+3 M k_{0}^{2}\right) \sinh 2 k_{0} h+2 k_{0} h\left(1-\varepsilon K+M k_{0}^{2}\right)}\right]
$$

By a similar technique, the integral involving $\cos \mu t$ in (4.1) becomes for large $t$

$$
-2 i M \pi \sin \sigma t\left[\frac{B\left(r, k_{0}\right) D\left(k_{0}\right) \mu\left(k_{0}\right)\left(1+\varepsilon K+M k_{0}^{2}\right) \cosh k_{0}(h-y)}{\left(1+M k_{0}^{2}\right)\left\{\left(1-\varepsilon K+3 M k_{0}^{2}\right) \sinh 2 k_{0} h+2 k_{0} h\left(1-\varepsilon K+M k_{0}^{2}\right)\right\}}\right]
$$

We thus finally obtain $\varphi$ for large $t$ as

$$
\left.\begin{array}{c}
\varphi(r, y, t) \sim-\frac{1}{\pi i} \int_{0}^{\infty}\left[\frac{g a(\xi) \sin \sigma t}{\xi D(\xi)}\left\{\frac{\xi\left(1-\varepsilon K+M \xi^{2}\right) \cosh \xi y-K \sinh \xi y}{\mu^{2}-\sigma^{2}}\right\}\right. \\
-\frac{g T_{0} \pi \sinh \xi h}{\sigma D(\xi)} \cos \sigma t
\end{array}\right\} \begin{gathered}
\left\{\frac{\xi\left(1-\varepsilon K+M \xi^{2}\right)(\cosh \xi y-1)-K(\sinh \xi y-\operatorname{coth} \xi h)}{\mu^{2}-\sigma^{2}}\right\} \\
\left.+\frac{T_{0} \pi}{\sigma}(\cosh \xi y-1)\right] B(r, \xi) d \xi \\
-2 i \frac{B\left(r, k_{0}\right)\left(1-\varepsilon K+M k_{0}^{2}\right) \cosh k_{0}(h-y)}{\Delta_{0}} \\
\cdot\left[a\left(k_{0}\right) \cos \sigma t+M \pi \sin \sigma t \frac{D\left(k_{0}\right) \mu\left(k_{0}\right)}{1+M k_{0}^{2}}\right]
\end{gathered}
$$

where

$$
\Delta_{0} \equiv\left(1-\varepsilon K+3 M k_{0}^{2}\right) \sinh 2 k_{0} h+2 k_{0} h\left(1-\varepsilon K+M k_{0}^{2}\right)
$$

For the evaluation of the integrals in (4.7), we rotate the contour involving the second kind Hankel functions clockwise by $\pi$ and noting that

$$
\frac{H_{0}^{(2)}\left(r \xi e^{-i \pi}\right)}{H_{1}^{(2)}\left(a \xi e^{-i \pi}\right)}=-\frac{H_{0}^{(1)}(r \xi)}{H_{1}^{(1)}(a \xi)}
$$


(4.7) can then be written as

$$
\begin{gathered}
\varphi(r, y ; t) \sim-\frac{1}{\pi i} \int_{-\infty}^{\infty}\left[\frac{g a(\xi)}{\xi D(\xi)} \sin \sigma t\left\{\frac{\left.\xi\left(1-\varepsilon K+M \xi^{2}\right) \cosh \xi y-K \sinh \xi y\right)}{\mu^{2}-\sigma^{2}}\right\}\right. \\
-\frac{g T_{0} \pi \sinh \xi h}{\sigma D(\xi)} \cos \sigma t \\
\cdot\left\{\frac{\xi\left(1-\varepsilon K+M \xi^{2}\right)(\cosh \xi y-1)-K(\sinh \xi y-\operatorname{coth} \xi h)}{\mu^{2}-\sigma^{2}}\right\} \\
\left.+\frac{T_{0} \pi}{\sigma}(\cosh \xi y-1)\right] \frac{H_{0}^{(1)}(r \xi)}{H_{1}^{(1)}(a \xi)} d \xi \\
-2 i \frac{B\left(r, k_{0}\right)\left(1-\varepsilon K+M k_{0}^{2}\right) \cosh k_{0}(h-y)}{\Delta_{0}} \\
\cdot\left[a\left(k_{0}\right) \cos \sigma t+M \pi \sin \sigma t \frac{D\left(k_{0}\right) \mu\left(k_{0}\right)}{1+M k_{0}^{2}}\right] .
\end{gathered}
$$

Making $h \rightarrow \infty$, the result given in [7] for $\sigma<(g / \varepsilon)^{1 / 2}$ can be deduced from (4.9). The contour integrals in (4.9) can now be extended to the contour $\Gamma$, the whole real axis with indentations above the pole at $\xi=-k_{0}$ and below the pole at $\xi=k_{0}$. Then this may be evaluated by the method of residues at the poles $\xi= \pm k_{0}$ and at $\xi=i k_{n}(n=1,2, \ldots)$ after enclosing it by a semicircle of large radius in the upper half of the complex $\xi$-plane.

Thus we obtain $\varphi$ after simplifications as

$$
\begin{aligned}
\varphi(r, y, t) \sim-4 \sum_{n=1}^{\infty} \frac{K_{0}\left(r k_{n}\right)}{K_{1}\left(a k_{n}\right)} \frac{\cos k_{n}(h-y)}{\Delta_{n}} \\
\quad \times\left\{A_{n}\left(1-\varepsilon K-M k_{n}^{2}\right) \sin \sigma t-M \pi \sigma \cos k_{n} h \cdot \cos \sigma t\right\} \\
-4 \frac{\cosh k_{0}(h-y)}{\Delta_{0} F}\left[a\left(k_{0}\right)\left(1-\varepsilon K+M k_{0}^{2}\right)(D \sin \sigma t+E \cos \sigma t)\right. \\
\left.-M \pi \sigma \cosh k_{0} h(D \cos \sigma t-E \sin \sigma t)\right]
\end{aligned}
$$

where

$$
\begin{gathered}
\Delta_{n} \equiv\left(1-\varepsilon K-3 M k_{n}^{2}\right) \sin 2 k_{n} h+2 k_{n} h\left(1-\varepsilon K-M k_{n}^{2}\right), \\
A_{n} \equiv \int_{0}^{h} \cos k_{n}(h-y) U(s) d s \\
F \equiv J_{1}^{2}\left(a k_{0}\right)+Y_{1}^{2}\left(a k_{0}\right) \\
D \equiv J_{0}\left(k_{0} r\right) J_{1}\left(k_{0} a\right)+Y_{0}\left(k_{0} r\right) Y_{1}\left(k_{0} a\right) \\
E \equiv J_{0}\left(k_{0} r\right) Y_{1}\left(k_{0} r\right)-J_{1}\left(k_{0} a\right) Y_{0}\left(k_{0} r\right)
\end{gathered}
$$


and $K_{n}$ 's $(n=0,1)$ are the modified Bessel functions of second kind. Using (4.10) in (3.11), we obtain the depression of the inertial surface for large time as

$$
\begin{aligned}
\zeta(r, t) \sim-\frac{4 \sigma}{g} \sum_{n=1}^{\infty} \frac{K_{0}\left(r k_{n}\right)}{K_{1}\left(a k_{n}\right)} \frac{\left(1-M k_{n}^{2}\right) \cos k_{n} h}{\Delta_{n}} \\
\times\left\{A_{n} \cos \sigma t+\frac{M \pi \sigma \cos k_{n} h}{1-\varepsilon K-M k_{n}^{2}} \sin \sigma t\right\} \\
-4 \frac{\sigma}{g} \frac{\cosh k_{0} h\left(1+M k_{0}^{2}\right)}{\Delta_{0} F}\left[a\left(k_{0}\right)(D \cos \sigma t-E \sin \sigma t)\right. \\
\left.+M \pi \sigma \frac{\cosh k_{0} h}{\left(1-\varepsilon K+M k_{0}^{2}\right)}(D \sin \sigma t+E \cos \sigma t)\right] .
\end{aligned}
$$

As $r \rightarrow \infty$ this becomes

$$
\begin{array}{r}
\zeta(r, t) \sim\left[a ( k _ { 0 } ) \left\{J_{1}\left(k_{0} a\right) \cos \left(k_{0} r-\sigma t-\pi / 4\right)\right.\right. \\
\left.+Y_{1}\left(k_{0} a\right) \sin \left(k_{0} r-\sigma t-\pi / 4\right)\right\} \\
+\left\{Y_{0}\left(k_{0} a\right) \cos \left(k_{0} r-\sigma t-\pi / 4\right)\right. \\
\left.-J_{1}\left(k_{0} a\right) \sin \left(k_{0} r-\sigma t-\pi / 4\right)\right\} \\
\left.\cdot M \pi \sigma \frac{\cosh k_{0} h}{\left(1-\varepsilon K+M k_{0}^{2}\right)}\right] \\
+\left[-\frac{2^{5 / 2}}{\left(\pi k_{0} r\right)^{1 / 2}} \frac{\sigma\left(1+M k_{0}^{2}\right)}{g \Delta_{0} F}\right]
\end{array}
$$

which represent outgoing progressive waves. In the absence of the surface tension, (4.17) coincides with the corresponding result given in [8] (of course for $\left.\sigma<(g / \varepsilon)^{1 / 2}\right)$.

\section{Conclusion}

The velocity potential as well as the depression of the inertial surface in the problem of vertical cylindrical wave-maker in a liquid of uniform finite depth are derived when the effect of surface tension is included. In the absence of the inertial surface, (4.10) can be identified with the result given in (6.3) of [9] if we put $\mu=i \sigma$ there and take the imaginary part of (6.3) in [9] after multiplying by $e^{-i \sigma t}$. In the absence of surface tension and inertial surface, 
results given in [5] can also be recovered from (4.10).

\section{Acknowledgement}

The author thanks Prof. B. N. Mandal, Indian Statistical Institute, Calcutta, for his guidance in the preparation of this paper and the referees for some useful suggestions. He also thanks the University Grants Commission, New Delhi for the award of a teacher fellowship (grant no. F-8-34/986(D11)).

\section{References}

[1] D. V. Evans, "The influence of surface tension on the reflection of water waves by a plane vertical barrier", Proc. Camb. Phil. Soc. 64 (1968) 795-810.

[2] D. V. Evans, "The effect of surface tension on the waves produced by a heaving circular cylinder", Proc. Camb. Phil. Soc. 64 (1968) 833-847.

[3] T. H. Havelock, "Forced surface waves on water", Phil. Mag. 8 (1929) 569-576.

[4] L. M. Hocking, "Capillary-gravity waves produced by a heaving body", J. Fluid Mech. 186 (1988) 337-349.

[5] B. N. Mandal, "A note on the Havelock's cylindrical wave-maker problems", Rev. Roum. Sci. Techn.-Mec. Appl. 34 (1989) 185-190.

[6] B. N. Mandal and K. Kundu, "A note on the singularities in the theory of water waves with an inertial surfacen, J. Austral. Math. Soc. Ser. B28 (1986) 271-278.

[7] B. N. Mandal and K. Kundu, "A note on the cylindrical wavemaker problem in a liquid with an inertial surface," Int. J. Engng. Sci. 27 (1989) 393-398.

[8] B. N. Mandal and K. Kundu, "A cylindrical wave-maker problem in a liquid of finite depth with an inertial surface", Indian J. Pure and Appl. Math. 20 (1989) 505-512.

[9] P. F. Rhodes-Robinson, "On the forced surface waves due to a vertical wave-maker in the presence of surface tension", Proc. Camb. Phil. Soc. 70 (1971) 323-337.

[10] P. F. Rhodes-Robinson, "On the generation of water waves at an inertial surface," $J$. Austral. Math. Soc. Ser. B25 (1984) 366-383.

[11] P. F. Rhodes-Robinson, "Note on the effect of surface tension on water waves at an inertial surface”, J. Fluid Mech. 125 (1982) 375-377.

[12] E. C. Titchmarsh, Eigenfunction expansions associated with second order differential equations, (Oxford University Press, 1962, p. 87). 